\title{
Image inpainting via variational approximation of a Dirichlet problem with free discontinuity
}

\author{
Michele Carriero, Antonio Leaci and Franco Tomarelli \\ Communicated by Frank Duzaar
}

\begin{abstract}
We study the variational approximation of an inpainting model for 2-dimensional images which are locally damaged. The scheme provides $\Gamma$-convergence of elliptic functionals to a Dirichlet problem with free discontinuity and free gradient discontinuity.
\end{abstract}

Keywords. Calculus of variations, free discontinuity problems, image segmentation and inpainting.

2010 Mathematics Subject Classification. 49K10, 49K20, 49J45.

\section{Introduction}

In this paper we refine the variational model for inpainting of 2-D monochromatic images which was introduced in $[25,27]$ and we study its approximation by showing the $\Gamma$-convergence of a suitable sequence of elliptic functionals. Moreover we describe a numerical algorithm which we apply to the inpainting of some model images. The limit functional and the approximating functionals are defined by suitable penalizations of the Dirichlet datum for a second order free discontinuity problem. Hence the results strongly depend on the regularity results proven in [22].

In image restoration the term inpainting denotes the process of filling in the missing information over subdomains where a given image is damaged: these domains may correspond to scratches in a camera picture, occlusion by objects, blotches in an old movie film or aging of canvas and colors in a painting $([5,28$, 29, 34, 43]).

Minimization of Blake \& Zisserman functional is a variational approach to segmentation and denoising in image analysis which deals with free discontinuity, free gradient discontinuity and second derivatives. This second order functional was introduced in [8] to avoid over-segmentation of steep gradients (ramp effect) and other drawbacks which occur in lower order models as in case of Mumford \& Shah

This work is supported by PRIN 2008 M.I.U.R (Progetto "Problemi variazionali con scale multiple"). 
functional ([41]). We refer to $[8,15-17,19,21,37,40]$ for motivation and analysis of variational approach to image segmentation and digital image processing.

First order variational approach to image segmentation and inpainting based on free discontinuities favours straight edges and produces artificial corners (see [29] and [34]), while the presence of second derivatives smooths such corners in the Blake \& Zisserman approach (see Figure 3 below).

About minimization of the Blake \& Zisserman functional under Neumann boundary condition we refer to $[14-16,18,20]$. For a description of the rich list of differential, integral and geometric extremality conditions we refer to [21]. The results of the paper [22] were deeply exploited in [23] and [26] to study fine properties of local minimizers of Blake \& Zisserman functional under Neumann boundary condition, particularly about their singular set related to optimal segmentation; in the present paper they are applied to the derivation and the study of a variational approximation for image inpainting.

In general uniqueness of minimizers for functionals of this kind fails due to lack of convexity. We refer to [9] for explicit examples of multiplicity. Nevertheless in the 1-D formulation, uniqueness of minimizer is a generic property with respect to admissible data: in [10] is proven that for a $G_{\delta}$ (countable intersection of dense open sets) set of admissible data the minimizer is unique. Hence the whole picture is coherent with the presence of instable patterns, each of them corresponding to a bifurcation of optimal segmentation under variation of parameters related to contrast threshold, "luminance sensitivity", resistance to noise, crease detection, double edge detection.

Here we introduce two second order functionals $E$ and

$$
F=E+\mu \int_{\Omega \backslash U}|v-g|^{2} d \mathbf{x}
$$

where $g$ is a given $L^{\infty}$ datum with $0 \leq g \leq 1$ (see (1.1), (2.13)), aiming to image inpainting, respectively in the cases of complete or partial loss of information in a small subregion $\Omega$ (see Figures 1 and 2 below), then we define the related weak functionals $\mathcal{E}, \mathcal{F}$ (see (4.5) and (5.4)) and their approximations, denoted respectively by $\mathcal{E}_{k}$ and $\mathscr{F}_{k}$.

The functional $E$ is defined as follows:

$$
\begin{array}{r}
E\left(K_{0}, K_{1}, v\right)=\int_{\widetilde{\Omega} \backslash\left(K_{0} \cup K_{1}\right)}\left(\left|D^{2} v(\mathbf{x})\right|^{2}+\delta(v(\mathbf{x})-1 / 2)^{2}\right) d \mathbf{x} \\
+\alpha \mathscr{H}^{1}\left(K_{0} \cap \widetilde{\Omega}\right)+\beta \mathscr{H}^{1}\left(K_{1} \cap \widetilde{\Omega}\right) .
\end{array}
$$

Here $\widetilde{\Omega}$ denotes an open set, which represents the image domain, with $\widetilde{\Omega} \subset \subset \mathbb{R}^{2}$, $0<\beta \leq \alpha \leq 2 \beta$ and $\delta>0$. The integral of $(v-1 / 2)^{2}$, weighted with a fixed 
positive constant $\delta$, penalizes deviation of gray levels in the output from the central gray level in the raw image and it entails the coerciveness of $E$.

Let $\Omega$ be an open set with $\Omega \subset \subset \widetilde{\Omega}$ and $\partial \Omega$ Lipschitz, and let $w$ denote a given $L^{\infty}(\widetilde{\Omega} \backslash \bar{\Omega})$ function with $0 \leq w \leq 1$, representing the gray level intensity of the raw image under processing, which is damaged due to the presence of blotches in the set $\bar{\Omega}$ : the noiseless intensity $w$ is known in $\widetilde{\Omega} \backslash \bar{\Omega}$ while is completely lost in the possibly disconnected set $\Omega$.

To face the inpainting problem for $w$, we look for minimizers of $E$ among triplets $\left(K_{0}, K_{1}, v\right)$ which fulfill the Dirichlet condition

$$
v=w \quad \text { a.e. on } \widetilde{\Omega} \backslash \bar{\Omega} \text {. }
$$

and are essential triplets, say they fulfill the following properties:

$$
\left\{\begin{array}{l}
K_{0}, K_{1} \text { are Borel subsets of } \mathbb{R}^{2}, \quad K_{0} \cap K_{1}=\emptyset, \\
K_{0} \cup K_{1} \text { is the smallest closed set such that } v \in C^{2}\left(\widetilde{\Omega} \backslash\left(K_{0} \cup K_{1}\right)\right), \\
\text { and } v \text { is approximately continuous in } \widetilde{\Omega} \backslash K_{0} .
\end{array}\right.
$$

The notion of essential triplet selects those triplets which are cleansed of every spurious artifact that does not affect the functional value and are good representatives in equivalence classes of admissible triplets, which allows highly irregular gray level function $v$ : see Remark 2.5 and Lemmas 2.6, 2.7 below, for comparison with [23, Definition 2.1] of admissible triplets.

If $\left(K_{0}, K_{1}, u\right)$ is an essential minimizing triplet of $E$ fulfilling Dirichlet condition (1.2), then $u$ provides the inpainted restoration of the whole image, and $K_{0} \cup K_{1}$ can be interpreted as an optimal segmentation of the restored image: the three elements of a minimizing triplet $\left(K_{0}, K_{1}, u\right)$ play respectively the role of edges, creases and smoothly varying intensity in the region $\widetilde{\Omega} \backslash\left(K_{0} \cup K_{1}\right)$ for the segmented image.

To achieve the existence of minimizing triplets of $E$, following a general approach introduced by De Giorgi and Ambrosio in the seminal papers [30,31], we introduce a relaxed functional: the weak Blake $\&$ Zisserman functional for inpainting (see $\mathfrak{F}(v)$ in (3.3) and also $\mathfrak{F}(v)$ in (3.9)). The idea is to deal with a simpler functional, which depends on the function $v$ alone, and to recover the sets $K_{0}$ (jump points of $v$ ) and $K_{1}$ (crease points of $v$ ) by taking respectively the discontinuity sets $\overline{S_{v}}$ and $\overline{S_{\nabla v}} \backslash S_{v}$. The functional class where we set the problem is given by second order generalized functions with special bounded variation: say $\operatorname{GSBV}^{2}(\widetilde{\Omega})$ (for the formal definition see (3.1) and (3.2)). The class $\operatorname{GSBV}^{2}(\widetilde{\Omega})$ is the right functional setting, more appropriate than $\mathrm{BH}(\widetilde{\Omega})$ (bounded hessian functions whose second derivatives are Radon measure). Indeed $\mathrm{BH}$ functions in 
two variables are continuous with integrable gradient; on the other hand $\mathrm{BH}$ contains too much irregular functions: for instance the choice of a raw image as a primitive of the Cantor-Vitali function leads to inf $E=0$ in $\mathrm{BH}(\widetilde{\Omega})$.

In this framework compactness and lower semicontinuity [15, Theorems 8,10$]$ give the existence of minimizers for the relaxed functional $\mathfrak{S}(v)$. The results of Theorem 2.1 are achieved by showing partial regularity of the obtained weak solution with penalized Dirichlet datum (Theorem 3.1).

The approximation algorithms to deal with the two terms $\mathscr{H}^{1}\left(K_{0} \cap \widetilde{\Omega}\right)$ and $\mathscr{H}^{1}\left(K_{1} \cap \widetilde{\Omega}\right)$ are technically quite difficult. Moreover, for the functional (3.3) for inpainting, the approach developed in [1] must be suitably adapted, in order to approximate it with a sequence of (simpler) functionals not involving lineic energies.

An important problem is the one of finding effective numerical methods suitable for the determination of the solutions given in Theorems 2.1 and 2.2. The appropriate framework and techniques to solve this problem are provided by the $\Gamma$-convergence theory [33].

Variational approximation of Blake \& Zisserman functional aimed to image segmentation and denoising under Neumann boundary condition has been studied in [1] and [12] based on $\Gamma$-convergence of elliptic functionals; while a different approach based on a finite difference scheme for the approximation of the integral functional is proposed and studied in [11].

Conversely, here we propose a variational approximation of Blake \& Zisserman functional aimed to image inpainting under Dirichlet boundary condition, by defining a suitable sequence of $\Gamma$-convergent elliptic functionals $\mathcal{E}_{k}$ (see (4.6)). Moreover in the last section we describe a numerical algorithm based on finite differences to solve the Euler-Lagrange system of the approximating functionals.

There are three main differences with respect to the variational approximation studied by Ambrosio, Faina \& March in [1]: first, here is present a Dirichlet-type boundary condition, which is penalized by the term

$$
k \int_{\widetilde{\Omega} \backslash \Omega}|v-w|^{2} d \mathbf{x}
$$

in the approximating functionals $\mathcal{E}_{k}$ and is exactly recovered in the $\Gamma$ limit $\mathcal{E}$ (see (4.5), (4.6)); second, here the image domain is the set $\widetilde{\Omega}$ while in the paper [1] it is the set $\Omega$, nevertheless in both cases the image is processed only in the region $\Omega$; third, in the present context we lack also a noisy image defined in the whole $\widetilde{\Omega}$, which would allow to prescribe a fidelity term of the kind

$$
\mu \int_{\widetilde{\Omega}}|v-g|^{2} d \mathbf{x}
$$

this fact entails a lack of summability for $v$ and it is compensated by the addition 
of the term

$$
\delta \int_{\widetilde{\Omega} \backslash\left(K_{0} \cup K_{1}\right)}(v-1 / 2)^{2} d \mathbf{x} .
$$

The outline of the paper is the following. The strong formulation of the inpainting model is introduced in Section 2. The weak formulation of the inpainting model is introduced in Section 3. Several estimates on approximating functionals $\varepsilon_{k}$ are collected in Section 4 . Statements and proofs about variational convergence are given in Sections 5, 6 and 7; the analysis of the case $\alpha=\beta$ is complete, thanks to the results proven in [26] (uniform density estimates for minimizers and the coincidence of Hausdorff measure and Minkowski content of their singular set); the case $\alpha \neq \beta$ requires an additional technical assumption in order to achieve the $\Gamma$-lim sup inequality. Some numerically inpainted output of images partially occluded by masks are shown in Section 8.

The novelty of the present paper is the use of Blake \& Zisserman functional to tackle the inpainting problem and its formulation in terms of essential triplets. The main results are Theorems 2.1, 2.2 about existence of strong minimizers, Theorems 5.3, 5.4, whose proofs strongly rely on the approach of [1], and the numerical experiments, described in Section 8.

\section{Strong Blake \& Zisserman functional for image inpainting}

In this section we present the strong formulation of the problem and the existence of its solutions by stating two main theorems.

The first one is Theorem 2.1 below, which is a general tool since it deals both with discontinuity and gradient discontinuity in the raw image $w$ which is given in $\widetilde{\Omega} \backslash \bar{\Omega}$ and must be processed in $\Omega$. Without loss of generality we can assume that the raw image $w$ can be always extended with constant value equal to $1 / 2$ in $\bar{\Omega}$.

Theorem 2.1 (Second order variational model for image inpainting: minimization of functional $E$ ). Assume $\alpha, \beta, \delta, \Omega, \widetilde{\Omega}$ and $w: \widetilde{\Omega} \rightarrow[0,1]$ fulfil:

$$
\Omega \subset \subset \widetilde{\Omega} \subset \subset \mathbb{R}^{2},
$$

$\Omega$ Lipschitz open set with piece-wise $C^{2}$ boundary, $\widetilde{\Omega}$ open set,

$$
0<\beta \leq \alpha \leq 2 \beta, \quad \delta>0,
$$

$\left(T_{0}, T_{1}, w\right)$ essential triplet, $\quad T_{0} \cup T_{1}$ is a finite union of $C^{1}$ curves,

$$
\begin{gathered}
\mathscr{H}^{1}\left(\left(T_{0} \cup T_{1}\right) \cap \widetilde{\Omega}\right)<+\infty, \quad\left(T_{0} \cup T_{1}\right) \cap \partial \Omega \text { is a finite set, } \\
D^{2} w \in L^{\infty}\left(\widetilde{\Omega} \backslash\left(T_{0} \cup T_{1} \cup \partial \Omega\right)\right) .
\end{gathered}
$$


Then there exists a triplet $\left(P_{0}, P_{1}, u\right)$ which minimizes the functional $E$ defined by (1.1) among triplets fulfilling (1.2) and (1.3), with $E\left(P_{0}, P_{1}, u\right)<+\infty$.

Moreover any triplet $\left(K_{0}, K_{1}, v\right)$ which minimizes the functional $E$ among triplets fulfilling (1.2) and (1.3), verifies:

$$
\begin{gathered}
K_{0} \cap \widetilde{\Omega} \text { and } K_{1} \cap \widetilde{\Omega} \text { are }\left(\mathscr{H}^{1}, 1\right) \text { rectifiable sets, } \\
\mathscr{H}^{1}\left(K_{0} \cap \widetilde{\Omega}\right)=\mathscr{H}^{1}\left(\overline{S_{v}}\right), \quad \mathscr{H}^{1}\left(K_{1} \cap \widetilde{\Omega}\right)=\mathscr{H}^{1}\left(\overline{S_{\nabla v}} \backslash S_{v}\right),
\end{gathered}
$$

$\left\{\begin{array}{l}v \in \operatorname{GSBV}^{2}(\widetilde{\Omega}) \cap L^{2}(\widetilde{\Omega}), \text { hence } \\ v \text { and } \nabla v \text { have well-defined two-sided traces, finite } \mathscr{H}^{1} \text { a.e. on } K_{0} \cup K_{1},\end{array}\right.$

where $S_{v}$ and $S_{\nabla v}$ respectively denote the singular sets of $v$ and $\nabla v$ (see Section 3).

The second statement of this section is Theorem 2.2 below. It deals with free discontinuity and free gradient discontinuity in $\widetilde{\Omega} \backslash \Omega$ of the given raw image $w$ to be processed together with some additional noisy information, denoted by $g$, in a Borel subset $\Omega \backslash U$ with

$$
U \subset \subset \Omega \subset \subset \widetilde{\Omega} .
$$

Theorem 2.2 refers to the second model proposed in this paper. Such functional is labeled by $F$ and deals with the noisy part by summing the fidelity term

$$
\int_{\Omega \backslash U}|v-g|^{2} d \mathbf{x}
$$

to the functional $E$. Precisely, we introduce the functional

$$
F\left(K_{0}, K_{1}, v\right)=E\left(K_{0}, K_{1}, v\right)+\mu \int_{\Omega \backslash U}|v-g|^{2} d \mathbf{x}
$$

to be minimized over essential triplets $\left(K_{0}, K_{1}, v\right)$ fulfilling the Dirichlet condition (1.2).

Theorem 2.2 (Second order variational model for image inpainting: minimization of functional $F$ ). Assume $\widetilde{\Omega} \subset \subset \mathbb{R}^{2}$ is an open set, $\alpha, \beta, \delta, \Omega$ and $w$ fulfil (2.3)(2.6) and

$$
\begin{gathered}
\mu>0, \quad g \in L^{\infty}(\Omega \backslash U), \quad 0 \leq g \leq 1, \\
\left\{\begin{array}{l}
U \subset \subset \Omega \subset \subset \widetilde{\Omega} \subset \subset \mathbb{R}^{2}, \\
U, \Omega \text { Lipschitz open sets with piece-wise } C^{2} \text { boundary. }
\end{array}\right.
\end{gathered}
$$


Then there exists a triplet $\left(P_{0}, P_{1}, u\right)$ which minimizes the functional

$$
\begin{aligned}
F\left(K_{0}, K_{1}, v\right)= & E\left(K_{0}, K_{1}, v\right)+\mu \int_{\Omega \backslash U}|v-g|^{2} d \mathbf{x} \\
= & \int_{\widetilde{\Omega} \backslash\left(K_{0} \cup K_{1}\right)}\left(\left|D^{2} v\right|^{2}+\delta|v-1 / 2|^{2}\right) d \mathbf{x} \\
& +\mu \int_{\Omega \backslash U}|v-g|^{2} d \mathbf{x} \\
& +\alpha \mathscr{H}^{1}\left(K_{0} \cap \widetilde{\Omega}\right)+\beta \mathscr{H}^{1}\left(K_{1} \cap \widetilde{\Omega}\right)
\end{aligned}
$$

among triplets fulfilling (1.2) and (1.3), with $F\left(P_{0}, P_{1}, u\right)<+\infty$.

Moreover any minimizing triplet $\left(K_{0}, K_{1}, v\right)$ fulfils (2.7)-(2.9).

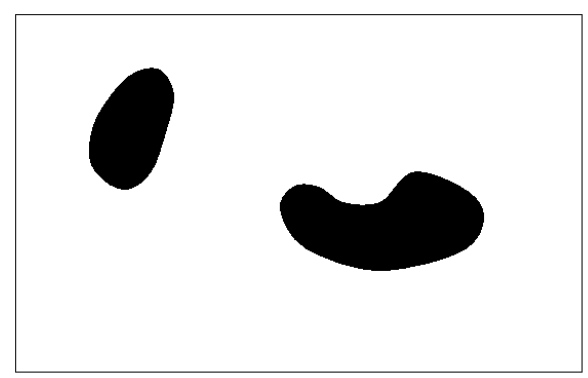

Figure 1. The image domain is the rectangle $\widetilde{\Omega}$. The blotches $\Omega \subset \subset \widetilde{\Omega}$ with complete loss of information are represented by the black region $\bar{\Omega}$ (see Theorem 2.1: minimization of functional $E$ ).

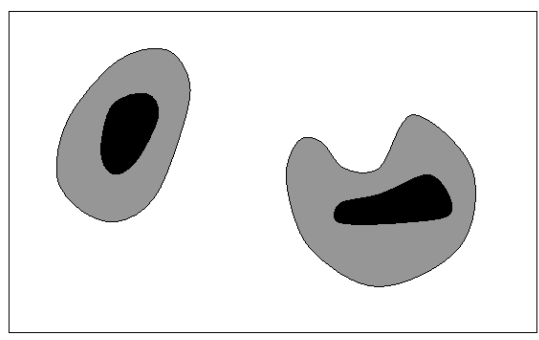

Figure 2. The image domain is the rectangle $\widetilde{\Omega}$. The blotches $\Omega \subset \subset \widetilde{\Omega}$ correspond to some loss of information: complete loss in the black region $U$, partially damaged image in the gray region $\Omega \backslash U$ (see Theorem 2.2: minimization of functional $F$ ). 
Remark 2.3. If $\left(K_{0}, K_{1}, v\right)$ is a minimizing triplet of $E$, then $v$ fulfills the Euler equation

$$
\Delta^{2} v+\delta(v-1 / 2)=0 \quad \text { in } \mathscr{D}^{\prime}\left(\Omega \backslash\left(K_{0} \cup K_{1}\right)\right),
$$

together with many integral and geometric relationships similar to the ones fulfilled by minimizing triplets of Blake \& Zisserman functional for image segmentation (see [21,23]).

Remark 2.4. We emphasize that the Dirichlet datum on the boundary $\partial \Omega$ is prescribed only by penalization, through $v=w$ a.e. on $\widetilde{\Omega} \backslash \bar{\Omega}$. Hence part of the segmentation may appear also on $\partial \Omega$ : when this phenomenon takes place, it is described by energy contribution of a length-type term $\mathscr{H}^{1}\left(\left(K_{0} \cup K_{1}\right) \cap \partial \Omega\right)$.

Remark 2.5. Obviously any essential triplet $\left(K_{0}, K_{1}, v\right)$ (e.g. a triplet fulfilling (1.3)) which minimizes $E$ with finite energy $E\left(K_{0}, K_{1}, v\right)<+\infty$, has a Lebesgue measurable third element $v$, since $v$ belongs to $C^{2}\left(\widetilde{\Omega} \backslash\left(K_{0} \cup K_{1}\right)\right)$ and since we have $\mathscr{L}^{2}\left(K_{0} \cup K_{1}\right)=0$.

Referring to Theorems 2.1 and 2.2, we discuss a comparison between the definition (1.3) of essential triplets given here and the definition of admissible triplets and of essential minimizing triplets given in [23, 25, 27]. This is achieved by Remark 2.5 and by Lemmas 2.6 and 2.7 below.

Lemma 2.6. The definition (1.3) selects a representative in each equivalence class of admissible triplets, with respect to the following equivalence relationship:

$$
\begin{aligned}
& \left(T_{0}, T_{1}, v\right) \sim\left(P_{0}, P_{1}, z\right) \\
& \Longleftrightarrow\left\{v=z \text { a.e. on } \widetilde{\Omega}, \mathscr{H}^{1}\left(T_{0} \triangle P_{0}\right)=\mathscr{H}^{1}\left(T_{1} \triangle P_{1}\right)=0\right\} .
\end{aligned}
$$

Moreover, any triplet fulfilling (1.3) satisfies also

$$
\begin{gathered}
v \text { is measurable in } \widetilde{\Omega}, \\
K_{1}=\bar{K}_{1} \backslash K_{0}, \quad K_{0}=\bar{K}_{0} \backslash K_{1} .
\end{gathered}
$$

Proof. The properties 2.15 and 2.16 follow by [26, Lemma 2.12].

Lemma 2.7. Theorems 2.1 and 2.2 refer to the minimization of functionals

$$
E=E_{b}+E_{\ell} \quad \text { and } \quad F=E+\mu \int_{\Omega \backslash U}|v-g|^{2} d \mathbf{x}
$$


where $E_{b}$ and $E_{\ell}$ denote respectively the bulk energy and the lineic energy

$$
\begin{aligned}
E_{b} & =\int_{\widetilde{\Omega} \backslash\left(K_{0} \cup K_{1}\right)}\left(\left|D^{2} v\right|^{2}+\delta|v-1 / 2|^{2}\right) d \mathbf{x}, \\
E_{\ell} & =\alpha \mathscr{H}^{1}\left(K_{0} \cap \widetilde{\Omega}\right)+\beta \mathscr{H}^{1}\left(K_{1} \cap \widetilde{\Omega}\right) \\
& =(\alpha-\beta) \mathscr{H}^{1}\left(K_{0} \cap \widetilde{\Omega}\right)+\beta \mathscr{H}^{1}\left(\left(K_{0} \cup K_{1}\right) \cap \widetilde{\Omega}\right) .
\end{aligned}
$$

The papers $[16,22,23]$ focused the analysis on the minimization with Dirichlet boundary conditions over admissible triplets (see [23, Definition 2.1]) of

$$
\widetilde{E}=E_{b}+\widetilde{E}_{\ell} \text { and } \widetilde{F}=\widetilde{E}+\mu \int_{\Omega \backslash U}|v-g|^{2} d \mathbf{x}
$$

where $\widetilde{E}_{\ell}=\alpha \mathscr{H}^{1}\left(K_{0} \cap \widetilde{\Omega}\right)+\beta \mathscr{H}^{1}\left(\left(K_{1} \backslash K_{0}\right) \cap \widetilde{\Omega}\right)$. Then:

(i) the present minimization of $E$ over essential triplets fulfilling condition (1.2) is equivalent to the minimization of $\widetilde{E}$ over admissible triplets (see [23, Definition 2.1]), say it provides an essential minimizing triplet in the sense of [26, Definition 2.6],

(ii) the present minimization of $F$ over essential triplets fulfilling condition (1.2) is equivalent to the minimization of $\widetilde{F}$ over admissible triplets.

The same statements (i) and (ii) hold true for the Neumann boundary conditions, i.e. when constraint (1.2) is dropped everywhere.

Proof. Obviously any essential triplet is an admissible triplet. For any admissible triplet $\left(U_{0}, U_{1}, u\right)$ we define another triplet $\left(K_{0}, K_{1}, v\right)=\mathscr{P}\left(U_{0}, U_{1}, u\right)$ as follows:

$$
v=\widetilde{u}
$$

$K$ is the smallest closed subset of $U_{0} \cup U_{1}$ such that $\widetilde{u} \in C^{2}(\widetilde{\Omega} \backslash K)$,

$$
\begin{aligned}
& K_{0}=\overline{U_{0} \cap K} \backslash\left(U_{1} \backslash U_{0}\right), \\
& K_{1}=\overline{U_{1} \cap K} \backslash U_{0} .
\end{aligned}
$$

Then, thanks to [26, Theorem 2.12], we have:

(I) $\mathcal{P}\left(K_{0}, K_{1}, v\right)$ is an essential triplet for any admissible triplet $\left(K_{0}, K_{1}, v\right)$;

(II) $E\left(\mathcal{P}\left(K_{0}, K_{1}, v\right)\right)=E\left(K_{0}, K_{1}, v\right)$ for any triplet $\left(K_{0}, K_{1}, v\right)$ which minimizes $E$ over admissible triplets,

(III) $E\left(\mathcal{P}\left(K_{0}, K_{1}, v\right)\right) \leq E\left(K_{0}, K_{1}, v\right)$ for any admissible triplet $\left(K_{0}, K_{1}, v\right)$,

(IV) $\mathcal{P}^{2}\left(K_{0}, K_{1}, v\right)=\mathscr{P}\left(K_{0}, K_{1}, v\right)$ for any admissible triplet, 
hence we get the equivalence (i) about minimization of $E$ over essential triplets and minimization of $\widetilde{E}$ over admissible triplets.

The same discussion proves the equivalence (ii) about minimization of $F$ and $\widetilde{F}$, since the difference refers only to the lineic part $E_{\ell}$ and $\widetilde{E}_{\ell}$ of the functionals.

Proof of Theorems 2.1 and 2.2. We follow exactly the same procedure of the papers [25, 27]: we apply the direct methods of Calculus of Variations to functionals (1.1) and (2.13), by proving the partial regularity for solutions of a weak version $\mathcal{E}$ of (1.1) and $\mathscr{F}$ of (2.13), which are introduced respectively in (3.3) and (3.9) of Section 3.

Remark 2.8. For the sake of simplicity the regularity assumption on the Dirichlet datum has been formulated by (2.4)-(2.6).

We emphasize that all Theorems 2.1, 2.2, 5.3 and 5.4 hold true if the assumptions (2.4) and (2.6) respectively are substituted by the following much more general assumptions, which allow to deal with highly irregular raw images:

$$
\begin{aligned}
& \left\{\begin{array}{l}
\left(T_{0}, T_{1}, w\right) \text { essential triplet, } \\
\exists \varepsilon>0, \varrho_{0}>0 \text { such that } \mathscr{H}^{1}\left(\left(T_{0} \cup T_{1}\right) \cap B_{\varrho}(\mathbf{x}) \cap(\widetilde{\Omega} \backslash \bar{\Omega})\right) \geq \varepsilon \varrho \\
\quad \forall \mathbf{x} \in\left(T_{0} \cup T_{1}\right) \cap \partial \Omega, \forall \varrho \leq \varrho_{0},
\end{array}\right. \\
& \left\{\begin{array}{l}
D^{2} w \in L^{2}\left(\widetilde{\Omega} \backslash\left(T_{0} \cup T_{1}\right)\right), \quad D^{2} w \in L^{\infty}\left(A \backslash \left(T_{0} \cup T\right.\right. \\
\text { there exists } A \text { open set such that } \partial \Omega \subset A \subset \widetilde{\Omega}, \\
\exists C>0:\|\nabla w\|_{L^{\infty},\left\|\nabla^{2} w\right\|_{L^{\infty}} \leq C \text { in } A,} \\
\operatorname{Lip}\left(\gamma^{\prime}\right) \leq C \text { with } \gamma \text { arc-length parametrization of } \partial \Omega, \\
\exists \bar{\varrho}>0: \mathscr{H}^{1}\left(\partial \Omega \cap B_{\varrho}(\mathbf{x})\right)<C \varrho \quad \forall \mathbf{x} \in \partial \Omega, \forall \varrho \leq \bar{\varrho} .
\end{array}\right.
\end{aligned}
$$

\section{Weak Blake \& Zisserman functional for image inpainting}

We denote by $B_{\varrho}(\mathbf{x})$ the open ball $\left\{\mathbf{y} \in \mathbb{R}^{2}:|\mathbf{y}-\mathbf{x}|<\varrho\right\}$, and set

$$
B_{\varrho}=B_{\varrho}(\mathbf{0}), \quad B_{\varrho}^{+}=B_{\varrho} \cap\{(x, y): y>0\}, \quad B_{\varrho}^{-}=B_{\varrho} \cap\{(x, y): y<0\} .
$$

We denote by $\chi_{V}$ the characteristic function of $V$ for any $V \subset \mathbb{R}^{2}$, by $\mathscr{H}^{1}(V)$ its 1-dimensional Hausdorff measure and by $|V|$ its Lebesgue outer measure.

For any Borel function $v: \Omega \rightarrow \mathbb{R}$ and $\mathbf{x} \in \Omega, z \in \overline{\mathbb{R}}:=\mathbb{R} \cup\{-\infty,+\infty\}$, we

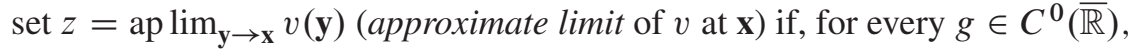

$$
g(z)=\lim _{\varrho \rightarrow 0} f_{B_{\varrho}(\mathbf{0})} g(v(\mathbf{x}+\boldsymbol{\xi})) d \xi
$$


the function $\widetilde{v}(\mathbf{x})=$ ap $\lim _{\mathbf{y} \rightarrow \mathbf{x}} v(\mathbf{y})$ is called representative of $v$; the singular set of $v$ is

$$
S_{v}=\left\{\mathbf{x} \in \Omega: \nexists z \text { such that } \operatorname{ap}_{\mathbf{y} \rightarrow \mathbf{x}} \lim v(\mathbf{y})=z\right\} .
$$

A Borel function $v: \Omega \rightarrow \mathbb{R}$ is approximately continuous at $\mathbf{x} \in \Omega$ if and only if $v(\mathbf{x})=\operatorname{ap} \lim _{\mathbf{y} \rightarrow \mathbf{x}} v(\mathbf{y})$.

By referring to $[2,16,21,36], D v$ denotes the distributional gradient of $v, \nabla v(\mathbf{x})$ denotes the approximate gradient of $v$, say $v$ is approximately differentiable at $\mathbf{x}$ if there exists a vector $\nabla v(\mathbf{x}) \in \mathbb{R}^{2}$ (the approximate gradient of $v$ at $\mathbf{x}$ ) such that

$$
\underset{\mathbf{y} \rightarrow \mathbf{x}}{\operatorname{ap} \lim } \frac{|v(\mathbf{y})-\widetilde{v}(\mathbf{x})-\nabla v(\mathbf{x}) \cdot(\mathbf{y}-\mathbf{x})|}{|\mathbf{y}-\mathbf{x}|}=0 .
$$

A function $u \in \mathrm{BV}(\Omega)$ is approximately differentiable a.e. and for $\mathscr{H}^{1}$ almost all $\mathbf{x} \in S_{u}$ there exist $v(\mathbf{x}) \in \partial B_{1}, v_{+}(\mathbf{x}) \in \mathbb{R}, v_{-}(\mathbf{x}) \in \mathbb{R}$ with $v_{+}(\mathbf{x})>v_{-}(\mathbf{x})$ such that

$$
\begin{aligned}
& \lim _{\varrho \rightarrow 0} \varrho^{-2} \int_{\left\{\mathbf{y} \in B_{\varrho}: \mathbf{y} \cdot v(\mathbf{x})>0\right\}}\left|v(\mathbf{x}+\mathbf{y})-v_{+}(\mathbf{x})\right| d \mathbf{y}=0, \\
& \lim _{\varrho \rightarrow 0} \varrho^{-2} \int_{\left\{\mathbf{y} \in B_{\varrho}: \mathbf{y} \cdot v(\mathbf{x})<0\right\}}\left|v(\mathbf{x}+\mathbf{y})-v_{-}(\mathbf{x})\right| d \mathbf{y}=0 .
\end{aligned}
$$

$\operatorname{By} \operatorname{SBV}(\Omega)$ we denote the De Giorgi class of functions $v \in \mathrm{BV}(\Omega)$ such that

$$
\int_{\Omega}|D v|=\int_{\Omega}|\nabla v| d \mathbf{x}+\int_{S_{v}}\left|v^{+}-v^{-}\right| d \mathscr{H}^{1} .
$$

Moreover,

$$
\begin{aligned}
\operatorname{SBV}_{\text {loc }}(\Omega) & :=\left\{v: v \in \operatorname{SBV}\left(\Omega^{\prime}\right) \forall \Omega^{\prime} \subset \subset \Omega\right\}, \\
\operatorname{GSBV}(\Omega) & :=\left\{v: \Omega \rightarrow \mathbb{R} \text { Borel }:-k \vee v \wedge k \in \operatorname{SBV}_{\text {loc }}(\Omega) \forall k \in \mathbb{N}\right\}, \\
\operatorname{GSBV}^{2}(\Omega) & :=\left\{v \in \operatorname{GSBV}(\Omega): \nabla v \in(\operatorname{GSBV}(\Omega))^{2}\right\} .
\end{aligned}
$$

If $v \in \operatorname{GSBV}(\Omega)$, then $\nabla v$ exists a.e., and for $v \in \operatorname{GSBV}^{2}(\Omega)$ we set

$$
\nabla^{2} v=\nabla(\nabla v)
$$

With the aim of minimizing functional $E$, we introduce the weak Blake $\&$ Zisserman functional for inpainting $\mathfrak{F}$, which is similar to the one introduced in [15] for image segmentation,

$$
\mathfrak{F}(v):= \begin{cases}\int_{\widetilde{\Omega}}\left(\left|\nabla^{2} v\right|^{2}+\delta(v-1 / 2)^{2}\right) d \mathbf{x} & \\ \quad+(\alpha-\beta) \mathscr{H}^{1}\left(S_{v}\right)+\beta \mathscr{H}^{1}\left(S_{v} \cup S_{\nabla v}\right) & \text { if } v \in X(\widetilde{\Omega}), \\ +\infty & \text { otherwise, }\end{cases}
$$


where

$$
X(\widetilde{\Omega}) \stackrel{\text { def }}{=}\left\{v \in \operatorname{GSBV}^{2}(\widetilde{\Omega}) \cap L^{2}(\widetilde{\Omega}): v=w \text { a.e. on } \widetilde{\Omega} \backslash \bar{\Omega}\right\} .
$$

We emphasize that $\mathfrak{E}$ is still a nonconvex functional but has the advantage of depending only on the function $v$ and not on the sets $K_{0}, K_{1}$; nevertheless the optimal sets $K_{0}, K_{1}$ can be recovered by suitable transformations of the singular sets $S_{v}$ and $S_{\nabla v}$.

Theorem 3.1 (Weak second order variational model for image inpainting: minimization of functional (5). Assume (2.1)-(2.3) and $w: \widetilde{\Omega} \rightarrow[0,1]$ such that $w \in C^{2}\left(\widetilde{\Omega} \backslash \overline{\left(S_{w} \cup S_{\nabla w}\right)}\right), \quad$ w approximately continuous in $\widetilde{\Omega} \backslash S_{w}$,

$$
\begin{gathered}
\mathfrak{S}(w)<+\infty \\
\mathscr{H}^{1}\left(\overline{\left(S_{w} \cup S_{\nabla w}\right)} \backslash\left(S_{w} \cup S_{\nabla w}\right)\right)=0, \\
\mathscr{H}^{1}\left(\overline{\left(S_{w} \cup S_{\nabla w}\right)} \cap \partial \Omega\right)=0 \quad\left(\text { or } \overline{\left(S_{w} \cup S_{\nabla w}\right)} \cap \partial \Omega \text { finite set }\right) .
\end{gathered}
$$

Then there exists $v$ minimizing the functional $\mathfrak{S}$ in $X(\widetilde{\Omega})$ with finite energy.

Proof. Obviously $\mathfrak{E}(v) \geq 0$ for all $v \in X(\widetilde{\Omega})$. Assumptions (3.5)-(3.8), the interpolation Theorem 6 in [15] and [32, Lemma 2.3] entail

$$
w \in X(\widetilde{\Omega}) \text { and } \inf _{v \in X(\widetilde{\Omega})} \mathfrak{E}(v)<+\infty .
$$

Let $v_{k} \in X(\widetilde{\Omega})$ be a minimizing sequence for $\mathfrak{5}$. By [15, Theorem 8] there is $v_{\infty}$ in $X(\widetilde{\Omega})$ and a subsequence such that, without relabeling, $v_{k} \rightarrow v_{\infty}$ a.e. on $\widetilde{\Omega}$.

The property $v_{k}=w$ in $\widetilde{\Omega} \backslash \bar{\Omega}$ entails $v_{\infty}=w$ in $\widetilde{\Omega} \backslash \bar{\Omega}$. By [15, Theorem 10],

$$
\mathfrak{S}\left(v_{\infty}\right) \leq \liminf _{k} \mathfrak{E}\left(v_{k}\right),
$$

hence $\mathfrak{E}\left(v_{\infty}\right)=\inf _{v \in X(\widetilde{\Omega})} \mathfrak{E}(v)$.

Analogously, in order to study the functional $F$, we introduce the weak Blake $\&$ Zisserman functional for inpainting $\mathfrak{F}$, which is similar to the one introduced in [15] for image segmentation,

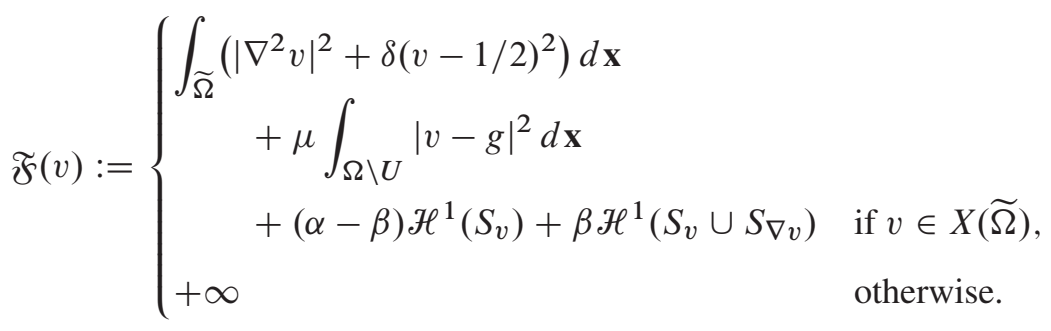


Theorem 3.2 (Weak second order variational model for image inpainting: minimization of functional $\mathfrak{F}$ ). Assume (2.1)-(2.3), (3.5), (3.7), (3.8) and

$$
\mathfrak{F}(w)<+\infty .
$$

Then there exists $u$ minimizing the functional $\mathfrak{F}$ in $X(\widetilde{\Omega})$ with finite energy.

By Theorems 3.1 and 3.2 and by the regularity theory developed in [22, 25,27], we achieve the proofs of Theorems 2.1 and 2.2.

\section{Functional framework and approximating functionals}

In order to approximate the weak functional $\mathfrak{E}=\mathfrak{E}(v)$ we introduce a functional $\mathcal{E}=\mathcal{E}(v, s, \sigma)$ which depends also on the control functions $s, \sigma$ and coincides with $\mathfrak{S}$ for trivial choices ( $s \equiv 1, \sigma \equiv 1$ ) of the control functions. We define the spaces

$$
\begin{aligned}
& Y(\widetilde{\Omega}):=\left(\operatorname{GSBV}^{2}(\widetilde{\Omega}) \cap L^{2}(\widetilde{\Omega})\right) \times L^{\infty}(\widetilde{\Omega} ;[0,1]) \times L^{\infty}(\widetilde{\Omega} ;[0,1]), \\
& \mathcal{D}(\widetilde{\Omega}):=H^{2}(\widetilde{\Omega}) \times H^{1}(\widetilde{\Omega} ;[0,1]) \times H^{1}(\widetilde{\Omega} ;[0,1]) \subset Y(\widetilde{\Omega})
\end{aligned}
$$

and also the space, larger than $\mathfrak{D}(\widetilde{\Omega})$,

$$
\mathfrak{D}_{0}(\widetilde{\Omega}):=\left\{(v, s, \sigma): v, s, \sigma \in H^{1}(\widetilde{\Omega}), \sigma \nabla v \in W^{1, p}\left(\widetilde{\Omega} ; \mathbb{R}^{2}\right)\right\} \subset Y(\widetilde{\Omega})
$$

where $p=2 \gamma /(\gamma+2)$ and $\gamma \geq 2$.

In the following they are briefly denoted respectively by $\mathfrak{D}$ and $\mathfrak{D}_{0}$.

If $v \in \mathfrak{D}_{0}$, the approximate differentiability of $v$ and of $\sigma \nabla v$ imply that $\nabla^{2} v$ exists a.e. in $\{\sigma>0\}$ and is given by

$$
\nabla^{2} v=\frac{\nabla(\sigma \nabla v)-\nabla \sigma \otimes \nabla v}{\sigma} .
$$

We also set $\nabla^{2} v=0$ in $\{\sigma=0\}$. We define a functional

$$
\mathcal{E}: Y(\widetilde{\Omega}) \rightarrow[0,+\infty]
$$

as follows:

$$
\mathcal{E}(v, s, \sigma):= \begin{cases}\mathfrak{S}(v) & \text { if } v \in X(\widetilde{\Omega}), s \equiv 1, \sigma \equiv 1 \text { in } \widetilde{\Omega}, \\ +\infty & \text { otherwise. }\end{cases}
$$

Obviously, by Theorem 3.1, there exists the minimum of $\mathcal{E}$ in $Y(\widetilde{\Omega})$. 
In order to obtain the variational approximation of the functional (4.5), we introduce the sequence of elliptic functionals $\varepsilon_{k}: Y(\widetilde{\Omega}) \rightarrow[0,+\infty]$ as follows:

$$
\mathbb{E}_{k}(v, s, \sigma):= \begin{cases}\int_{\widetilde{\Omega}}\left(\sigma^{2}+\kappa_{k}\right)\left|D^{2} v\right|^{2} d \mathbf{x} \\ \quad+\xi_{k} \int_{\widetilde{\Omega}}\left(s^{2}+\zeta_{k}\right)|D v|^{\gamma} d \mathbf{x} \\ \quad+(\alpha-\beta) \int_{\widetilde{\Omega}}\left(\frac{1}{k}|D s|^{2}+k \frac{(s-1)^{2}}{4}\right) d \mathbf{x} \\ +\beta \int_{\widetilde{\Omega}}\left(\frac{1}{k}|D \sigma|^{2}+k \frac{(\sigma-1)^{2}}{4}\right) d \mathbf{x} \\ +\delta \int_{\Omega}|v-1 / 2|^{2} d \mathbf{x} \\ +k \int_{\widetilde{\Omega} \backslash \Omega}|v-w|^{2} d \mathbf{x} \quad \text { if }(v, s, \sigma) \in \mathfrak{D} \text { and } \kappa_{k}>0, \\ & \text { or }(v, s, \sigma) \in \mathfrak{D}_{0} \text { and } \kappa_{k}=0, \\ +\infty & \text { otherwise, }\end{cases}
$$

for $\gamma \geq 2$ and suitable positive infinitesimals $\kappa_{k}, \xi_{k}, \zeta_{k}$.

Functionals $\varepsilon_{k}$ are to be minimized on triplets of functions $(v, s, \sigma)$. We emphasize that the minimization acts not only on the restored image $v$ but also on two auxiliary functions: $s$ which is a control function for $D v$ and $\sigma$ which is a control function of the Hessian of $v$. To understand heuristically why this approximation holds, we observe that if $\left(v_{k}, s_{k}, \sigma_{k}\right)$ is a sequence of minimizers of $\varepsilon_{k}$, then the function $s_{k}$ assumes value 1 where $v$ is continuous and it is close to 0 in a tubular neighborhood of discontinuity set $S_{v}$ of thickness $1 / k$. As $k \rightarrow+\infty$, this neighborhood shrinks and then, for $k$ large enough, $s_{k}$ yields an approximate representation of the jump set of $v$.

The function $\sigma_{k}$, instead, assumes values near 0 only in a tubular neighborhood of $S_{v} \cup S_{\nabla v}$ of thickness $1 / k$. As $k \rightarrow+\infty$, this neighborhood shrinks and $\sigma_{k}$ yields an approximate representation of the jump and crease sets of $v$. The last term in (4.6) forces $v$ to assume the value $w$ in $\widetilde{\Omega} \backslash \bar{\Omega}$. The following term of ModicaMortola type ([39]) is inserted in the functional, to approximate the Hausdorff measure of the singular sets

$$
\mathscr{E}_{k}(z):=\int_{\widetilde{\Omega}}\left(\frac{1}{k}|D z|^{2}+k \frac{(z-1)^{2}}{4}\right) d \mathbf{x} .
$$

For any $k \in \mathbb{N}$, we prove the properties of the functionals $\varepsilon_{k}$ which allow the use 
of the direct methods in Calculus of Variations to prove the existence of minimizers of $\varepsilon_{k}$ in $Y(\widetilde{\Omega})$.

Theorem 4.1 (Compactness of $\varepsilon_{k}$ sublevels). For any fixed $k \in \mathbb{N}$,

(i) for any positive infinitesimals $\kappa_{k}, \xi_{k}, \zeta_{k}, \gamma \geq 2$ and any sequence

$$
V_{n}=\left(v_{n}, s_{n}, \sigma_{n}\right) \in \mathfrak{D}
$$

such that

$$
\sup _{n} \varepsilon_{k}\left(V_{n}\right)<+\infty
$$

there exists a subsequence $V_{n_{j}}$ and $V=(v, s, \sigma) \in \mathfrak{D}$ such that

$$
\begin{aligned}
V_{n_{j}} & \rightarrow V & \text { in } L^{1}\left(\widetilde{\Omega} ; \mathbb{R}^{3}\right), \\
D v_{n_{j}} & \rightarrow \text { Dv } & \text { a.e. on } \widetilde{\Omega}, \\
s_{n_{j}} \rightarrow s, \quad \sigma_{n_{j}} & \rightarrow \sigma \quad & \text { weakly in } H^{1}(\widetilde{\Omega} ;[0,1]) .
\end{aligned}
$$

(ii) for any positive infinitesimals $\xi_{k}, \zeta_{k}, \kappa_{k}=0, \gamma>2$ and any sequence

$$
V_{n}=\left(v_{n}, s_{n}, \sigma_{n}\right) \in \mathfrak{D}_{0}
$$

such that

$$
\sup _{n} E_{k}\left(V_{n}\right)<+\infty
$$

there exists a subsequence $V_{n_{j}}$ and $V=(v, s, \sigma) \in \mathfrak{D}_{0}$ such that

$$
\begin{aligned}
V_{n_{j}} & \rightarrow V & & \text { in } L^{1}\left(\widetilde{\Omega} ; \mathbb{R}^{3}\right), \\
D v_{n_{j}} & \rightarrow D v & & \text { a.e. on }\{\sigma>0\}, \\
s_{n_{j}} \rightarrow s, \quad \sigma_{n_{j}} & \rightarrow \sigma & & \text { weakly in } H^{1}(\widetilde{\Omega} ;[0,1]) .
\end{aligned}
$$

Proof. The proof of (i) is a straightforward consequence of Rellich's theorem. For the proof of (ii) see [1, Theorem 5.1].

Theorem 4.2 (Lower semicontinuity of $\mathcal{E}_{k}$ ). For any fixed $k \in \mathbb{N}$, for any positive infinitesimals $\kappa_{k}, \xi_{k}, \zeta_{k}, \gamma \geq 2$ and any sequence $V_{n}=\left(v_{n}, s_{n}, \sigma_{n}\right) \in \mathfrak{D}$ such that

$$
V_{n} \rightarrow V \in \mathfrak{D} \quad \text { in } L^{1}\left(\widetilde{\Omega} ; \mathbb{R}^{3}\right),
$$

the following inequality holds true:

$$
\mathcal{E}_{k}(V) \leq \liminf _{n} \mathcal{E}_{k}\left(V_{n}\right) .
$$

If $\kappa_{k}=0$ and $\gamma>2$, for any positive infinitesimals $\xi_{k}, \zeta_{k}$, and any sequence $V_{n}=\left(v_{n}, s_{n}, \sigma_{n}\right) \in \mathfrak{D}_{0}$ such that (4.8) holds in $\mathfrak{D}_{0}$, inequality (4.9) holds true. 
Proof. It is not restrictive to assume that $\varepsilon_{k}\left(V_{n}\right)$ converges to a finite limit. Then by Theorem 4.1

$$
\begin{aligned}
D v_{n} & \rightarrow D v & \text { a.e. on } \widetilde{\Omega}, \\
s_{n} \rightarrow s, \quad \sigma_{n} & \rightarrow \sigma & \text { weakly in } H^{1}(\widetilde{\Omega} ;[0,1]) .
\end{aligned}
$$

By collecting (4.8)-(4.10),

$$
\begin{array}{cc}
\left(\sigma_{n}^{2}+\kappa_{k}\right)^{1 / 2} D^{2} v_{n} \rightarrow\left(\sigma^{2}+\kappa_{k}\right)^{1 / 2} D^{2} v & \text { weakly in } L^{2}\left(\widetilde{\Omega} ; \mathbb{R}^{2 \times 2}\right), \\
\left(s_{n}^{2}+\zeta_{k}\right)^{1 / 2} D v_{n} \rightarrow\left(s^{2}+\zeta_{k}\right)^{1 / 2} D v & \text { weakly in } L^{2}\left(\widetilde{\Omega} ; \mathbb{R}^{2}\right) .
\end{array}
$$

Then by Ioffe's lower semicontinuity theorem the first two terms in $\varepsilon_{k}$ are lower semicontinuous. Since the sequences $s_{n}$ and $\sigma_{n}$ are bounded in $H^{1}(\widetilde{\Omega} ;[0,1])$, they weakly converge respectively to $s, \sigma$ and therefore the terms $\boldsymbol{E}_{k}(s)$ and $\boldsymbol{E}_{k}(\sigma)$, defined in (4.7), are lower semicontinuous.

For the proof of the case $\kappa_{k}=0$ and $\gamma>2$, see [1, Theorem 5.2].

Under the two sets of assumptions in Theorems 4.1 and 4.2, the compactness and lower semicontinuity properties of $\mathcal{E}_{k}$ entail that the problem

$$
\min \left\{\mathcal{E}_{k}(v, s, \sigma)\right\}
$$

has at least one solution for any $k \in \mathbb{N}$ among either the triplets $(v, s, \sigma) \in \mathfrak{D}$ or $(v, s, \sigma) \in \mathfrak{D}_{0}$.

\section{Convergence of approximating functionals}

In this section we collect the statements about convergence properties for the approximating functionals $\mathcal{E}_{k}$ (see definitions (3.3), (3.4), (4.5), (4.6)). The results hold true under the assumptions (2.3)-(2.6); the proofs are postponed to Sections 6 and 7.

All these variational approximations are obtained in the framework of the notion of $\Gamma$-convergence, introduced by De Giorgi and Franzoni in [33], whose definition is recalled below for reader's convenience.

Let $Y(\widetilde{\Omega})$ be endowed with the topology of $L^{1}\left(\widetilde{\Omega} ; \mathbb{R}^{3}\right)$ and let

$$
\mathcal{E}, \mathcal{E}_{k}: Y(\widetilde{\Omega}) \rightarrow[0,+\infty]
$$

be the functionals defined above, we say that the sequence $\left(\varepsilon_{k}\right) \Gamma$-converges to $\mathcal{E}$ if and only if for any $(v, s, \sigma) \in Y(\widetilde{\Omega})$ the following two conditions are satisfied:

(i) for any sequence $\left(v_{k}, s_{k}, \sigma_{k}\right)$ in $Y(\widetilde{\Omega})$ converging in $L^{1}\left(\widetilde{\Omega} ; \mathbb{R}^{3}\right)$ to $(v, s, \sigma)$, then

$$
\mathcal{E}(v, s, \sigma) \leq \liminf _{k} \mathcal{E}_{k}\left(v_{k}, s_{k}, \sigma_{k}\right),
$$


(ii) there exists a sequence $\left(v_{k}, s_{k}, \sigma_{k}\right)$ converging in $L^{1}\left(\widetilde{\Omega} ; \mathbb{R}^{3}\right)$ to $(v, s, \sigma)$ such that

$$
\limsup _{k} \varepsilon_{k}\left(v_{k}, s_{k}, \sigma_{k}\right) \leq \mathcal{E}(v, s, \sigma) .
$$

The importance of this notion relies on the fact that it implies the convergence of minimizers of the approximating functionals $\varepsilon_{k}$ to minimizers of the limiting functional $\mathscr{E}$ under an equicoercivity assumption. Moreover the $\Gamma$-convergence is stable with respect to continuous perturbations.

First we state the lower inequality of $\Gamma$-convergence.

Theorem 5.1. Assume $\gamma \geq 2$ and either $\kappa_{k}>0$ or $\kappa_{k}=0, \zeta_{k}>0$ definitively, and assume that

$$
\lim _{k \rightarrow+\infty} k \xi_{k}=+\infty
$$

Then, for every triplet $(v, s, \sigma) \in Y(\widetilde{\Omega})$ and for every sequence $\left(v_{k}, s_{k}, \sigma_{k}\right) \in \mathfrak{D}$ (or in $\mathfrak{D}_{0}$ if $\left.\kappa_{k}=0\right)$ converging to $(v, s, \sigma)$ in $L^{1}\left(\widetilde{\Omega} ; \mathbb{R}^{3}\right)$ as $k \rightarrow+\infty$, we have

$$
\mathcal{E}(v, s, \sigma) \leq \liminf _{k \rightarrow+\infty} \mathcal{E}_{k}\left(v_{k}, s_{k}, \sigma_{k}\right) .
$$

Moreover, (5.1) can be replaced by the condition $\xi_{k} \geq 0$ in the case $\alpha=\beta$.

Then we state the equicoercivity of the sequence $\left(\varepsilon_{k}\right)$ under the same pair of assumptions on the infinitesimals $\kappa_{k}, \zeta_{k}, \xi_{k}$ made in Theorem 5.1.

Theorem 5.2. Assume either $\gamma \geq 2$ and $\kappa_{k}>0$ or $\gamma>2, \kappa_{k}=0$ and $\zeta_{k}>0$ definitively, and

$$
\lim _{k \rightarrow+\infty} k \xi_{k}=+\infty, \quad \sup _{k} \varepsilon_{k}\left(v_{k}, s_{k}, \sigma_{k}\right)<+\infty
$$

Then the sequence $\left(v_{k}, s_{k}, \sigma_{k}\right)$ is relatively compact in the $L^{1}\left(\widetilde{\Omega} ; \mathbb{R}^{3}\right)$ topology and any limit point is of the form $(v, 1,1)$ with $v \in X(\widetilde{\Omega})$.

Now we consider the upper inequality of $\Gamma$-convergence. We first state a full $\Gamma$-convergence result in the special case when $\alpha=\beta$. We recall that a domain $\widetilde{\Omega}$ is strictly star-shaped if there exists $x_{0} \in \widetilde{\Omega}$ such that $t\left(\widetilde{\Omega}-x_{0}\right)+x_{0} \subset \subset \widetilde{\Omega}$ for any $t \in[0,1)$.

Theorem 5.3. Assume that $\alpha=\beta, \gamma \geq 2$ and $\widetilde{\Omega}$ is strictly star-shaped. Assume that $\kappa_{k}>0$ and $\kappa_{k}=o\left(1 / k^{4}\right)$, while $\xi_{k}=\zeta_{k}=0$. Then:

(i) the sequence $\left(\mathcal{E}_{k}\right) \Gamma$-converges to $\mathcal{E}$ in the $L^{1}\left(\widetilde{\Omega} ; \mathbb{R}^{3}\right)$ topology as $k \rightarrow+\infty$,

(ii) if $\left(v_{k}, s_{k}, \sigma_{k}\right)$ minimizes $\varepsilon_{k}$, the sequence $\left(v_{k}, s_{k}, \sigma_{k}\right)$ is relatively compact in $L^{1}\left(\widetilde{\Omega} ; \mathbb{R}^{3}\right)$ and any limit point corresponds to a triplet $(v, 1,1)$ with $v$ minimizer of $\mathfrak{F}$ in $X(\widetilde{\Omega})$. 
Eventually we state the upper inequality of $\Gamma$-convergence in the more general situation $\alpha \neq \beta$, under the assumptions that $v \in L^{\infty}(\widetilde{\Omega}),|\nabla v| \in L^{\gamma}(\widetilde{\Omega})$ and that, for the sets $S_{v}$ and $S_{v} \cup S_{\nabla v}$, Hausdorff measure and Minkowski content coincide (see Theorem 5.4 below).

For every set $A \subset \mathbb{R}^{2}$ and every positive real number $\varrho$, we denote by $(A)_{\varrho}$ the open tubular neighborhood of $A$ with radius $\varrho$, that is

$$
(A)_{\varrho}=\left\{x \in \mathbb{R}^{2}: \operatorname{dist}(x, A)<\varrho\right\} .
$$

We define the Minkowski 1-dimensional upper and lower content of the set $A$, respectively, by

$$
\mathcal{M}^{*}(A)=\limsup _{\varrho \rightarrow 0^{+}} \frac{\left|(A)_{\varrho}\right|}{2 \varrho}, \quad \mathcal{M}_{*}(A)=\liminf _{\varrho \rightarrow 0^{+}} \frac{\left|(A)_{\varrho}\right|}{2 \varrho} .
$$

It can be shown (see [36, Section 3.2.39]) that $\left|(A)_{\varrho}\right| / \varrho$ converges to $2 \mathscr{H}^{1}(A)$ as $\varrho \rightarrow 0^{+}$for any compact subset $A$ of a $C^{1}$ curve. In particular, by inner approximation this implies

$$
\mathcal{M}_{*}(A) \geq \mathscr{H}^{1}(A)
$$

for any $v \in \operatorname{BV}(\widetilde{\Omega})$ and any Borel set $A \subset S_{v}$, because $\mathscr{H}^{1}$-almost all of $S_{v}$ can be covered by $C^{1}$ curves. The inequality $\mathcal{M}^{*}(A) \leq \mathscr{H}^{1}(A)$, which implies

$$
\lim _{\varrho \rightarrow 0^{+}} \frac{\left|(A)_{\varrho}\right|}{2 \varrho}=\mathscr{H}^{1}(A),
$$

holds under very mild regularity assumptions on the set $A$ (see [3]).

Theorem 5.4. Assume that $\alpha \neq \beta$ and $\widetilde{\Omega}$ is strictly star-shaped. Assume that $\gamma \geq 2, \kappa_{k}=0, \zeta_{k}>0$, $\xi_{k}$ satisfies (5.1) and $\xi_{k} \zeta_{k}=o\left(1 / k^{\gamma-1}\right)$. Then, for $\mathrm{ev}$ ery triplet $(v, s, \sigma) \in Y(\widetilde{\Omega})$ such that $v \in L^{\infty}(\widetilde{\Omega}),|\nabla v| \in L^{\gamma}(\widetilde{\Omega})$ and

$$
\mathcal{M}^{*}\left(S_{v}\right) \leq \mathscr{H}^{1}\left(S_{v}\right), \quad \mathcal{M}^{*}\left(S_{v} \cup S_{\nabla v}\right) \leq \mathscr{H}^{1}\left(S_{v} \cup S_{\nabla v}\right),
$$

there exist $\left(v_{k}, s_{k}, \sigma_{k}\right) \in \mathfrak{D}_{0}$ converging to $(v, s, \sigma)$ in $L^{1}\left(\widetilde{\Omega} ; \mathbb{R}^{3}\right)$ as $k \rightarrow+\infty$ such that

$$
\limsup _{k \rightarrow+\infty} \mathcal{E}_{k}\left(v_{k}, s_{k}, \sigma_{k}\right) \leq \mathcal{E}(v, s, \sigma) .
$$

Hence the sequence $\left(\mathcal{E}_{k}\right) \Gamma$-converges to $\mathcal{E}$ in the $L^{1}\left(\widetilde{\Omega} ; \mathbb{R}^{3}\right)$ topology; if $\left(v_{k}, s_{k}, \sigma_{k}\right)$ minimizes $\mathcal{E}_{k}$, then the sequence $\left(v_{k}, s_{k}, \sigma_{k}\right)$ is relatively compact in $L^{1}\left(\widetilde{\Omega} ; \mathbb{R}^{3}\right)$ and any limit point corresponds to a triplet $(v, 1,1)$ with $v$ minimizer of $\mathcal{E}$ in $X(\widetilde{\Omega})$. 
Remark 5.5. All the definitions and theorems of the present section aim to the approximation of $\mathcal{E}$ with the functionals $\mathcal{E}_{k}$.

Obviously all the definitions and theorems of the present section hold true for the approximation of the functional

$$
\mathcal{F}(v, s, \sigma)=\mathcal{E}(v, s, \sigma)+\mu \int_{\Omega \backslash U}|v-g|^{2} d \mathbf{x}
$$

by simply substituting $\mathcal{E}_{k}$ with

$$
\widetilde{F}_{k}=\mathcal{E}_{k}+\mu \int_{\Omega \backslash U}|v-g|^{2} d \mathbf{x}
$$

and adding (2.11), (2.12) to the assumptions.

\section{Lower inequality and equicoerciveness}

In this section we prove the lower inequality of $\Gamma$-convergence (5.2) and the equicoercivity of the family $\left(\varepsilon_{k}\right)$. In the following it will be convenient also to consider functionals depending on the domain of integration.

Proof of Theorem 5.1. Let $V_{k}=\left(v_{k}, s_{k}, \sigma_{k}\right) \in \mathfrak{D}$ and $V \in Y(\widetilde{\Omega})$ be such that $V_{k} \rightarrow V$ in $L^{1}\left(\widetilde{\Omega} ; \mathbb{R}^{3}\right)$ as $k \rightarrow+\infty$. We assume that

$$
+\infty>L=\liminf _{k \rightarrow+\infty} \mathcal{E}_{k}\left(v_{k}, s_{k}, \sigma_{k}\right)=\lim _{n \rightarrow+\infty} \mathcal{E}_{k_{n}}\left(v_{k_{n}}, s_{k_{n}}, \sigma_{k_{n}}\right),
$$

otherwise the result is trivial. For notational simplicity we set $V_{n}=\left(v_{k_{n}}, s_{k_{n}}, \sigma_{k_{n}}\right)$ and we assume that $V_{n}$ converge a.e. to $V=(v, s, \sigma)$ as $n \rightarrow+\infty$. Since $L$ is finite, it follows that, by Fatou's lemma, $v=w$ in $\widetilde{\Omega} \backslash \bar{\Omega}, s=\sigma=1$ in $\widetilde{\Omega}$. By [1, Theorem 3.1], we have

$$
\begin{aligned}
\mathscr{E}(v, s, \sigma, \widetilde{\Omega}) \leq \liminf _{n \rightarrow+\infty}\left(\int _ { \widetilde { \Omega } } \left(\sigma_{n}^{2}\right.\right. & \left.+\kappa_{n}\right)\left|D^{2} v_{n}\right|^{2} d \mathbf{x} \\
& +\xi_{n} \int_{\widetilde{\Omega}}\left(s_{n}^{2}+\zeta_{n}\right)\left|D v_{n}\right|^{\gamma} d \mathbf{x} \\
& \left.+(\alpha-\beta) \boldsymbol{E}_{n}\left(s_{n}\right)+\beta \boldsymbol{E}_{n}\left(\sigma_{n}\right)\right) \\
+ & \liminf _{n \rightarrow+\infty}\left(\delta \int_{\Omega}\left|v_{n}-1 / 2\right|^{2} d \mathbf{x}+n \int_{\widetilde{\Omega} \backslash \Omega}\left|v_{n}-w\right|^{2} d \mathbf{x}\right) \\
\leq & \liminf _{n \rightarrow+\infty} \mathcal{E}_{n}\left(v_{n}, s_{n}, \sigma_{n}\right) .
\end{aligned}
$$


Proof of Theorem 5.2. By reasoning as in the proof of [1, Theorem 3.1], by the equiboundedness of $\mathcal{E}_{k}\left(v_{k}, s_{k}, \sigma_{k}\right)$ it follows that $\left(s_{k}, \sigma_{k}\right) \rightarrow(1,1)$ in $L^{1}\left(\widetilde{\Omega} ; \mathbb{R}^{2}\right)$ as $k \rightarrow+\infty$ and there exists a subsequence (without relabeling) and measurable sets $C_{k} \subset \widetilde{\Omega}$ such that $\left|\widetilde{\Omega} \backslash C_{k}\right| \rightarrow 0$ and $u_{k}=v_{k} \chi C_{k}$ converge in $L^{1}(\widetilde{\Omega})$ to $v \in X(\widetilde{\Omega})$. Since $\left(v_{k}\right)$ is equibounded in $L^{2}(\widetilde{\Omega})$, by Hölder's inequality

$$
\left\|u_{k}-v_{k}\right\|_{L^{1}} \rightarrow 0 \quad \text { as } k \rightarrow+\infty,
$$

hence $v_{k} \rightarrow v$ in $L^{1}(\widetilde{\Omega})$.

\section{Upper inequality}

Proof of Theorem 5.3. Without loss of generality we can assume:

$$
v \in X(\widetilde{\Omega}), \quad\left|\nabla^{2} v\right| \in L^{2}(\widetilde{\Omega}), \quad \sigma \equiv 1 .
$$

Since we have that $\alpha=\beta$, we set $s_{k}=1$ for any $k$. As in [1], we can construct a sequence $v_{k}$ converging to $v$ in $L^{2}(\widetilde{\Omega})$, so that we can drop from the functionals $\varepsilon_{k}, \mathcal{E}$ the term

$$
\delta \int_{\Omega}|v-1 / 2|^{2} d x
$$

which is continuous with respect to the strong $L^{2}(\widetilde{\Omega})$ topology. Assuming that $\widetilde{\Omega}$ is strictly star-shaped with respect to the origin, we set

$$
\widetilde{\Omega}_{t}=t \widetilde{\Omega}, \quad \forall t \in(0,1) .
$$

By the assumption $\Omega \subset \subset \widetilde{\Omega}$, we have $\Omega \subset \widetilde{\Omega} t$, at least for $t$ near 1 . By the assumptions on $w$ and by assuming first that

$$
\mathcal{M}^{*}\left(S_{v} \cup S_{\nabla v}\right)=\mathscr{H}^{1}\left(S_{v} \cup S_{\nabla v}\right),
$$

following the construction of [1, Section 7.1], we obtain a sequence

$$
V_{k}=\left(v_{k}, 1, \sigma_{k}\right) \in \mathfrak{D}\left(\widetilde{\Omega}_{t}\right)
$$

such that

$$
\limsup _{k \rightarrow \infty} \mathcal{E}_{k}\left(v_{k}, 1, \sigma_{k}, \widetilde{\Omega}_{t}\right) \leq \mathcal{E}(v, 1,1, \widetilde{\Omega}) .
$$

We remark that the additional term

$$
k \int_{\widetilde{\Omega}_{t} \backslash \Omega}\left|v_{k}-w\right|^{2} d \mathbf{x}
$$


is infinitesimal, since $\left\|v_{k}\right\|_{L^{\infty}\left(\widetilde{\Omega}_{t} \backslash \Omega\right)} \leq\|w\|_{L^{\infty}(\widetilde{\Omega} \backslash \Omega)}$ and

$$
\lim _{k \rightarrow \infty} k\left|\left\{v_{k} \neq w\right\} \cap\left(\widetilde{\Omega}_{t} \backslash \Omega\right)\right|=0,
$$

as proved for (7.6) in [1], with $\varepsilon=1 / k$.

Then, the maps $V_{k, t}(x)=V_{k}(t x)$ belong to $\mathfrak{D}(\widetilde{\Omega})$ and satisfy

$$
\limsup _{k \rightarrow \infty} \varepsilon_{k}\left(v_{k}, 1, \sigma_{k}, \widetilde{\Omega}\right) \leq t^{-2} \mathcal{E}(v, 1,1, \widetilde{\Omega}),
$$

hence the desired sequence of the $\Gamma$-lim sup inequality can be constructed by a diagonal argument by letting $t \uparrow 1$.

The additional assumption (7.1) can be removed as follows. Consider for any $\lambda>0$ the penalized problem

$$
\min \left\{\int_{\widetilde{\Omega}}\left|\nabla^{2} u\right|^{2}+\lambda|u-v|^{2} d x+\beta \mathscr{H}^{1}\left(S_{u} \cup S_{\nabla u}\right): u \in X(\widetilde{\Omega})\right\},
$$

and let $u_{\lambda}$ be a minimizer (see [22]). Since $\mathfrak{S}\left(u_{\lambda}\right) \leq \mathfrak{F}(w)<+\infty$, it follows that $u_{\lambda} \rightarrow v$ in $L^{2}(\widetilde{\Omega})$ as $\lambda \rightarrow+\infty$. By the uniform density estimates on the singular set up to the boundary proved in [26, Theorem 5.9], any $u_{\lambda}$ fulfills (7.1), and therefore a sequence $V_{k}=\left(v_{k}, 1, \sigma_{k}\right)$ satisfying (7.2) can be obtained by a diagonal argument.

We remark that in the present case (say $\alpha=\beta$ ) the assumption (5.1) can be removed.

Claim (ii) follows from Theorem 5.2 and the properties of $\Gamma$-convergence.

Proof of Theorem 5.4. The proof is similar to that of [1, Theorem 3.4], taking into account (7.3).

\section{Numerical experiments}

In the present Section we describe a numerical approximation for the Euler-Lagrange system of equations of the functionals $\mathcal{E}_{k}$ in (4.6), which are $\Gamma$-convergent to Blake \& Zisserman functional for inpainting (4.5).

The approximation of Blake \& Zisserman functional by the $\Gamma$-convergence allows to approximate numerically the minimizers of the functionals $\varepsilon_{k}$ and consequently the minimizers of functional $\mathscr{E}$ too.

The approximation of second order functionals with free gradient discontinuity by elliptic functionals $\Gamma$-convergent has been stated and numerically implemented in the context of image segmentation and depth extraction from stereo images in [1], by adopting a discretization approach different from the one proposed 
here. For the sake of simplicity, we assume $\kappa_{k}=0$, and that the open set $\widetilde{\Omega}$ is a rectangle.

The system of Euler-Lagrange equations, associated to the $k$-th approximating functional $\mathcal{E}_{k}$ in (4.6), when the term $\kappa_{k}$ is neglected, is given by

$$
\left\{\begin{aligned}
\sigma^{2} \Delta^{2} v+2 \sigma & \left(2 D \sigma \cdot D(\Delta v)+v_{x x} \sigma_{x x}+2 v_{x y} \sigma_{x y}+v_{y y} \sigma_{y y}\right) \\
& +2\left(D^{2} v D \sigma\right) \cdot D \sigma-\xi_{k}\left(s^{2} \Delta v+2 s D v \cdot D s\right) \\
& +\delta\left(v-\frac{1}{2}\right) \chi_{\Omega}+k(v-w) \chi_{\widetilde{\Omega} \backslash \Omega}=0 \\
4(\alpha-\beta) \Delta s & =4 \xi_{k} k s|D v|^{2}+(\alpha-\beta) k^{2}(s-1) \\
4 \beta \Delta \sigma & =4 k \sigma\left|D^{2} v\right|^{2}+\beta k^{2}(\sigma-1)
\end{aligned}\right.
$$

in the open set $\widetilde{\Omega}$. For the sake of simplicity, in the following we could assume $\alpha=\beta$, hence $s \equiv 1$, and $\xi_{k}=0$, so that the system becomes a $2 \times 2$ system.

The system (8.1) can be discretized by finite central differences.

Let $h$ be fixed and let $\widetilde{\Omega}$ be the rectangle $(0, n+1) \times(0, m+1)$. Let $x_{i}=i h$ and $y_{j}=j h$ be a discretization of $\widetilde{\Omega}$ with step-size $h>0$ and let, for $i=1, \ldots, m$ and for $j=1, \ldots, n$,

$$
\left(\sigma_{i, j}\right)_{k}: \cong \sigma_{k}\left(x_{i}, y_{j}\right), \quad\left(u_{i, j}\right)_{k}: \cong u_{k}\left(x_{i}, y_{j}\right)
$$

be the approximate values of the solution $\left(u_{k}, \sigma_{k}\right)$ of (8.1) in the interior points of the discretization of $\widetilde{\Omega}$. On the boundary of $\widetilde{\Omega}$ we set $u=w$ and $\sigma=1$.

In the following we omit the index $k$ for the involved functions whenever this omission does not create any ambiguity.

We denote the finite difference approximations by the central differences of first partial derivatives of a given function $v$ as follows:

$$
\begin{aligned}
& v_{x}\left(x_{i}, y_{j}\right) \cong \frac{v_{i+1, j}-v_{i-1, j}}{2 h}, \\
& v_{y}\left(x_{i}, y_{j}\right) \cong \frac{v_{i, j+1}-v_{i, j-1}}{2 h},
\end{aligned}
$$

while the Laplace operator, $\Delta v:=v_{x x}+v_{y}$, is approximated by the 5-points scheme

$$
\frac{v_{i+1, j}+v_{i-1, j}-4 v_{i, j}+v_{i, j+1}+v_{i, j-1}}{h^{2}},
$$

where $v_{i \pm 1, j} \cong v\left(x_{i} \pm h, y_{j}\right)$ and $v_{i, j \pm 1} \cong v\left(x_{i}, y_{j} \pm h\right)$. 
The second partial derivatives are approximated by

$$
\begin{aligned}
& v_{x x}\left(x_{i}, y_{j}\right) \cong \frac{v_{i+1, j}-2 v_{i, j}+v_{i-1, j}}{h^{2}}, \\
& v_{y y}\left(x_{i}, y_{j}\right) \cong \frac{v_{i, j+1}-2 v_{i, j}+v_{i, j-1}}{h^{2}}, \\
& v_{x y}\left(x_{i}, y_{j}\right) \cong \frac{v_{i+1, j+1}-v_{i+1, j-1}-v_{i-1, j+1}+v_{i-1, j-1}}{4 h^{2}} .
\end{aligned}
$$

The approximations of the third partial derivatives in $\left(x_{i}, y_{j}\right)$ are

$$
\begin{aligned}
& v_{x x x} \cong \frac{v_{i+2, j}-2 v_{i+1, j}+2 v_{i-1, j}-v_{i-2, j}}{2 h^{3}}, \\
& v_{y y y} \cong \frac{v_{i, j+2}-2 v_{i, j+1}+2 v_{i, j-1}-v_{i, j-2}}{2 h^{3}}, \\
& v_{x y y} \cong \frac{v_{i+1, j+1}-2 v_{i+1, j}+v_{i+1, j-1}-v_{i-1, j+1}+2 v_{i-1, j}-v_{i-1, j-1}}{2 h^{3}}, \\
& v_{x x y} \cong \frac{v_{i+1, j+1}-v_{i+1, j-1}-2 v_{i, j+1}+2 v_{i, j-1}+v_{i-1, j+1}-v_{i-1, j-1}}{2 h^{3}} .
\end{aligned}
$$

The bilaplacian of $v, \Delta^{2} v:=v_{x x x x}+2 v_{x x y y}+v_{y y y y}$, is approximated by a 13-points scheme

$$
\begin{array}{r}
\frac{v_{i+2, j}+2 v_{i+1, j+1}-8 v_{i+1, j}+2 v_{i+1, j-1}+v_{i, j+2}-8 v_{i, j+1}+20 v_{i, j}}{h^{4}} \\
+\frac{-8 v_{i, j-1}+v_{i, j-2}+2 v_{i-1, j+1}-8 v_{i-1, j}+2 v_{i-1, j-1}+v_{i-2, j}}{h^{4}}
\end{array}
$$

By inserting the above schemes into the Euler-Lagrange equations, we obtain a numerical approximation for the system (8.1), which we used in the numerical experiments. The solution of the nonlinear algebraic system is approximated through a Gauss-Seidel relaxation method (see [12] for an analogous approach).

The stopping criteria for the iterative procedure are given by

$$
\frac{\| \sigma_{k}^{(p+1)}-\left.\sigma_{k}^{(p)}\right|_{2}}{\left\|\sigma_{k}^{(p+1)}\right\|_{2}} \leq \text { tol } \sigma_{\mathrm{GS}}
$$

and

$$
\frac{\left\|u_{k}^{(p+1)}-u_{k}^{(p)}\right\|_{2}}{\left\|u_{k}^{(p+1)}\right\|_{2}} \leq \text { tol } u_{\mathrm{GS}}
$$

where tol $\sigma_{\mathrm{GS}}$ and tol $u_{\mathrm{GS}}$ are fixed tolerances to control the relative errors on the approximations. Then, if $p^{*}$ is the iteration at which both inequalities are satisfied, the numerical solution of (8.1) is given by $\left(u_{k}^{\left(p^{*}\right)}, \sigma_{k}^{\left(p^{*}\right)}\right)$. 
In order to simulate the $\Gamma$-convergence in the discrete space, we generate a sequence of differential problems like (8.1) by changing the value of the parameter $k$ according to a suitable rule $k(I)$, where $I$ is the current iterate. Since we obtain the converge results for the $\Gamma$-convergence as $k \rightarrow+\infty$, in the discrete version we are interested in studying the numerical convergence of the sequence of discrete approximations, when $I$ increases.

To this end we use an iterative technique based on successive substitutions or Picard iterations (see [42]).

For $I=0$, we set $k(0)=0$ and we consider as initial guess for the Picard method $\left(u_{0}, \sigma_{0}\right)=\left(\mathbf{1}, w^{*}\right)$, that is $\sigma=1$ everywhere in $\widetilde{\Omega}$ and the function $u$ given by the datum $w$ in $\widetilde{\Omega} \backslash \Omega$ and by $1 / 2$ in $\Omega$.

For the first iteration, $I=1$, we set $k(1)=1$ and we calculate the numerical solution $\left(u_{1}^{\left(p^{*}\right)}, \sigma_{1}^{\left(p^{*}\right)}\right)$ of the system $(8.1)$ with $k=1$.

In general, for every $I$, let $\left(u_{k(I)}^{\left(p^{*}\right)}, \sigma_{k(I)}^{\left(p^{*}\right)}\right)$ be the numerical solution of the system (8.1) with $k=k(I)$ that is obtained by the convergence of the previous Gauss-Seidel relaxation algorithm.

In this context, to iterate by Picard method means that the initial approximation for the method used for solving system (8.1) with $k=k(I+1)$, is the numerical solution obtained by Gauss-Seidel method at the previous step, that is

$$
\left(u_{k(I+1)}^{(0)}, \sigma_{k(I+1)}^{(0)}\right)=\left(u_{k(I)}^{\left(p^{*}\right)}, \sigma_{k(I)}^{\left(p^{*}\right)}\right) \equiv\left(u_{k(I)}, \sigma_{k(I)}\right) .
$$

The Picard iterative procedure stops when the following criterion on relative errors is satisfied:

$$
\begin{aligned}
& (\operatorname{err} \sigma)_{k(I+1)}=\frac{\left\|\sigma_{k(I+1)}-\sigma_{k(I)}\right\|_{2}}{\left\|\sigma_{k(I+1)}\right\|_{2}} \leq \operatorname{err} \sigma, \\
& (\operatorname{err} u)_{k(I+1)}=\frac{\left\|u_{k(I+1)}-u_{k(I)}\right\|_{2}}{\left\|u_{k(I+1)}\right\|_{2}} \leq \operatorname{err} u
\end{aligned}
$$

where tol $\sigma$ and tol $u$ are fixed tolerances on $\sigma$ and $u$, respectively. In this case, we say that the numerical convergence has been achieved in $I^{*}=I+1$ number of iterations and the corresponding value of $k$ is given by $k^{*}=k\left(I^{*}\right)$.

We define the variation law $k(I)$ of the parameter $k$ in the following way:

$$
\left\{\begin{aligned}
k(0) & =0, \quad k(1)=1 \\
k(I+1) & =k(I)+\left\lceil\frac{(\operatorname{err} \sigma)_{k(I)}}{\operatorname{tol} \sigma}\right\rceil \text { if } I \geq 1
\end{aligned}\right.
$$

where $\lceil\cdot\rceil$ is the upper integer part. 
This strategy of variation for $k$ in each system (8.1) with $k=k(I)$ seems reasonable, because it is a good choice to increase $k$ quickly when large errors $(\operatorname{err} \sigma)_{k(I)}$ are present, while only a slow variation of $k$ is required when we are "near" to the requested tolerance on the error. In the numerical experiments we have chosen always $k=1$ as initial guess for the Picard method. We have considered the parameters $\alpha, \delta$ with values of the order $\alpha=10^{-4}, 10^{-5}$ and $\delta=1$.

We conclude by showing some pictures obtained in numerical experiments which exploit the variational approximation (4.6) of the functional (4.5): Figures 3, 4, 5 and 6, 7 where the inpainting algorithm removes masks or overlapping text (courtesy of G. Carboni and I. Sgura).
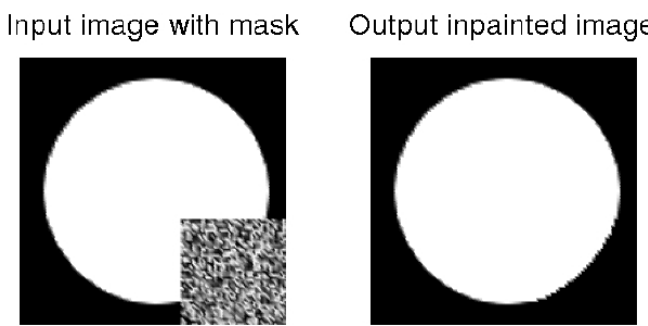

Jump segmentation

Figure 3. Inpainting of a circle without introducing artificial corners.

Input image with mask

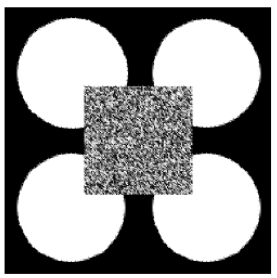

Output inpainted image

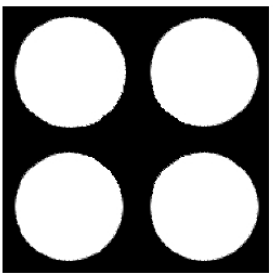

Jump segmentation

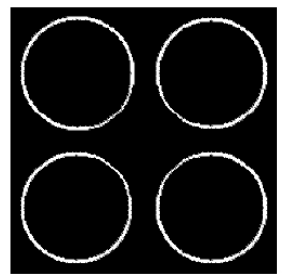

Figure 4. Inpainting of four circles.

Input image with mask

Output inpainted image

Jump segmentation
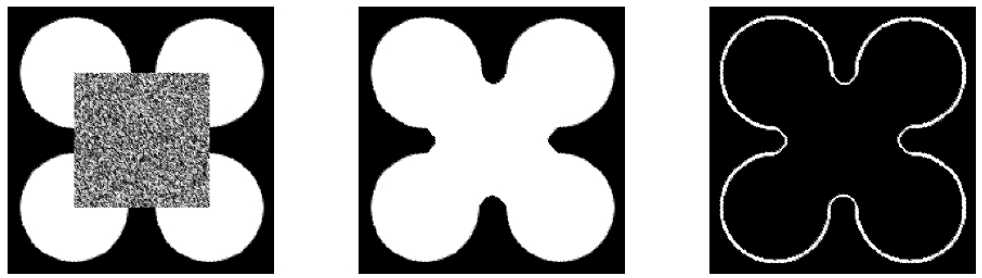

Figure 5. Inpainting of four circles with a big mask which gives connectivity. 
Input image with mask

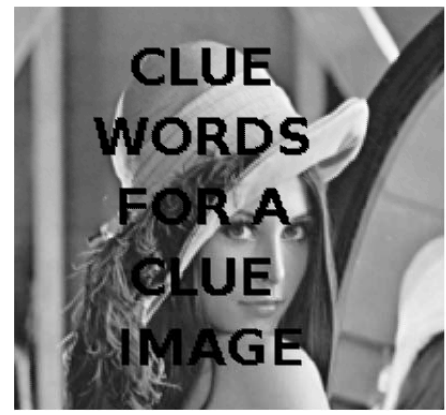

Output inpainted image

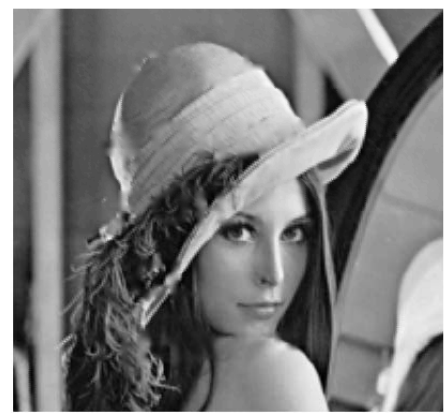

Figure 6. Text removal.

Input image with mask

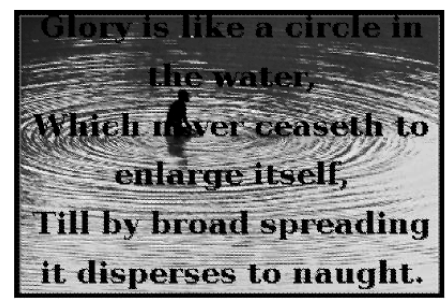

Output inpainted image

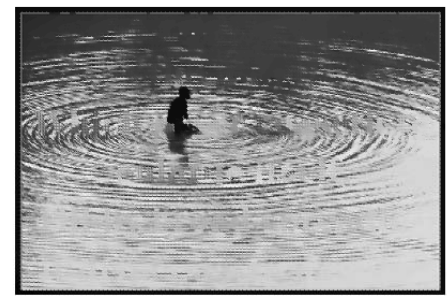

Figure 7. Text removal (from Shakespeare, "Henry VI").

\section{Bibliography}

[1] L. Ambrosio, L. Faina and R. March, Variational approximation of a second order free discontinuity problem in computer vision, SIAM J. Math. Anal. 32 (2001), 11711197.

[2] L. Ambrosio, N. Fusco and D. Pallara, Functions of Bounded Variation and Free Discontinuity Problems, Oxford Mathematical Monographs, Oxford University Press, Oxford, 2000.

[3] L. Ambrosio and V. M. Tortorelli, Approximation of functionals depending on jumps by elliptic functionals via $\Gamma$-convergence, Comm. Pure Appl. Math. 43 (1990), 9991036.

[4] L. Ambrosio and V. M. Tortorelli, On the approximation of free discontinuity problems, Boll. Unione Mat. Ital. B (7) 6 (1992), 105-123.

[5] G. Aubert and P. Kornprobst, Mathematical Problems in Image Processing, Partial Differential Equations and the Calculus of Variations, 2nd ed., Appl. Math. Sci. 147, Springer-Verlag, New York, 2006. 
[6] G. Bellettini and A. Coscia, Approximation of a functional depending on jumps and corners, Boll. Unione Mat. Ital. B (7) 8 (1994), 151-181.

[7] M. Bertalmío, V. Caselles, S. Masnou and G. Sapiro, Inpainting, in: Encyclopedia of Computer Vision, Springer-Verlag, Berlin (2011), 1-2.

[8] A. Blake and A. Zisserman, Visual Reconstruction, The MIT Press, Cambridge, 1987.

[9] T. Boccellari and F. Tomarelli, About well-posedness of optimal segmentation for Blake \& Zisserman functional, Ist. Lombardo Accad. Sci. Lett. Rend. A 142 (2008), 237-266.

[10] T. Boccellari and F. Tomarelli, Generic uniqueness of minimizer for Blake \& Zisserman functional, Rev. Mat. Complut. (2012), DOI 10.1007/s13163-012-0103-1.

[11] A. Braides, A. De Franceschi and E. Vitali, A compactness result for a second-order variational discrete model, ESAIM Math. Model. Numer. Anal. 46 (2012), 389-410.

[12] M. Carriero, A. Farina and I. Sgura, Image segmentation in the framework of free discontinuity problems, in: Calculus of Variations: Topics from the Mathematical Heritage of E. De Giorgi, Quad. Mat. 14, Dept. Math., Seconda Univ. Napoli, Caserta (2004), 86-133.

[13] M. Carriero and A. Leaci, Existence theorem for a Dirichlet problem with free discontinuity set, Nonlinear Anal. 15 (1990), no. 7, 661-677.

[14] M. Carriero, A. Leaci and F. Tomarelli, Free gradient discontinuities, in: Calculus of Variations, Homogeneization and Continuum Mechanics (Marseille 1993), Ser. Adv. Math. Appl. Sci. 18, World Scientific Publishing, River Edge (1994), 131-147.

[15] M. Carriero, A. Leaci and F. Tomarelli, A second order model in image segmentation: Blake \& Zisserman functional, in: Variational Methods for Discontinuous Structures (Como 1994), Progr. Nonlinear Differential Equations Appl. 25, Birkhäuser-Verlag, Basel (1996), 57-72.

[16] M. Carriero, A. Leaci and F. Tomarelli, Strong minimizers of Blake \& Zisserman functional, Ann. Sc. Norm. Super. Pisa Cl. Sci. (4) 25 (1997), no. 1-2, 257-285.

[17] M. Carriero, A. Leaci and F. Tomarelli, Density estimates and further properties of Blake \& Zisserman functional, in: From Convexity to Nonconvexity, Nonconvex Optim. Appl. 55, Kluwer Academics Dordrecht (2001), 381-392.

[18] M. Carriero, A. Leaci and F. Tomarelli, Necessary conditions for extremals of Blake \& Zisserman functional, C. R. Math. Acad. Sci. Paris 334 (2002), no. 4, 343-348.

[19] M. Carriero, A. Leaci and F. Tomarelli, Calculus of variations and image segmentation, J. Physiol. Paris 97 (2003), no. 2-3, 343-353.

[20] M. Carriero, A. Leaci and F. Tomarelli, Second order variational problems with free discontinuity and free gradient discontinuity, in: Calculus of Variations: Topics from the Mathematical Heritage of E. De Giorgi, Quad. Mat. 14, Dept. Math., Seconda Univ. Napoli, Caserta (2004), 135-186. 
[21] M. Carriero, A. Leaci and F. Tomarelli, Euler equations for Blake \& Zisserman functional, Calc. Var. Partial Differential Equations 32 (2008), no. 1, 81-110.

[22] M. Carriero, A. Leaci and F. Tomarelli, A Dirichlet problem with free gradient discontinuity, Adv. Math. Sci. Appl. 20 (2010), no. 1, 107-141.

[23] M. Carriero, A. Leaci and F. Tomarelli, A candidate local minimizer of Blake \& Zisserman functional, J. Math. Pures Appl. (9) 96 (2011), 58-87.

[24] M. Carriero, A. Leaci and F. Tomarelli, About Poincaré inequalities for functions lacking summability, Note Mat. 31 (2011) 67-85.

[25] M. Carriero, A. Leaci and F. Tomarelli, Free gradient discontinuity and image inpainting, Zap. Nauchn. Sem. S.-Peterburg. Otdel. Mat. Inst. Steklov. (POMI) 390 (2011), 92-116.

[26] M. Carriero, A. Leaci and F. Tomarelli, Uniform density estimates for Blake \& Zisserman functional, Discrete Contin. Dyn. Syst. Ser. A 31 (2011), no. 4, 1129-1150.

[27] M. Carriero, A. Leaci and F. Tomarelli, Free gradient discontinuity and image inpainting, J. Math. Sci. (N.Y.) 181 (2012), no. 6, 805-819.

[28] V. Caselles, G. Haro, G. Sapiro and J. Verdera, On geometric variational models for inpainting surface holes, Comput. Vis. Image Underst. 111 (2008), 351-373.

[29] T. F. Chan and J. Shen, Variational image inpainting, it Comm. Pure Appl. Math. 43 (2005), 579-619.

[30] E. De Giorgi, Free discontinuity problems in calculus of variations, in: Frontiers in Pure and Applied Mathematics, North-Holland, Amsterdam (1991), 55-61.

[31] E. De Giorgi and L. Ambrosio, Un nuovo tipo di funzionale del calcolo delle variazioni, Atti Accad. Naz. Lincei Cl. Sci. Fis. Mat. Natur. (8) 82 (1988), 199-210.

[32] E. De Giorgi, M. Carriero and A. Leaci, Existence theorem for a minimum problem with free discontinuity set, Arch. Ration. Mech. Anal. 108 (1989), 195-218.

[33] E. De Giorgi and T. Franzoni Su un tipo di convergenza variazionale, Atti Accad. Naz. Lincei Cl. Sci. Fis. Mat. Natur. (8) 58 (1975), no. 6, 842-850.

[34] S. Esedoglu and J.H. Shen, Digital inpainting based on the Mumford-Shah-Euler image model, European J. Appl. Math. 13 (2002), no. 4, 353-370.

[35] L. C. Evans and R.F. Gariepy, Measure Theory and Fine Properties of Functions, Stud. Adv. Math., CRC Press, Boca Raton, 1992.

[36] H. Federer, Geometric Measure Theory, Springer-Verlag, Berlin, 1969.

[37] N. Fusco, An Overview of the Mumford-Shah problem, Milan J. Math. 71 (2003) 95-119.

[38] F. A. Lops, F. Maddalena and S. Solimini, Hölder continuity conditions for the solvability of Dirichlet problems involving functionals with free discontinuities, Ann. Inst. H. Poincaré Anal. Non Linéaire 18 (2001), no. 6, 639-673. 
[39] L. Modica and S. Mortola, Un esempio di $\Gamma$-convergenza, Boll. Unione Mat. Ital. B (5) 14 (1977), 285-299.

[40] J. M. Morel and S. Solimini, Variational Models in Image Segmentation, Progr. Nonlinear Differential Equations Appl. 14, Birkhäuser-Verlag, Basel, 1995.

[41] D. Mumford and J. Shah, Optimal approximation by piecewise smooth functions and associated variational problems, Comm. Pure Appl. Math. 42 (1989), 577-685.

[42] J. Ortega and W. Rheinboldt, Iterative Solution of Nonlinear Equations in Several Variables, Academic Press, New York, 1970.

[43] J. Verdera, V. Caselles, M. Bertalmio and G. Sapiro, Inpainting surface holes, in: Proceedings of the 2003 International Conference on Image Processing. ICIP 2003 (Barcelona 2003), IEEE Press, Piscataway (2003), 903-906.

Received August 3, 2012; revised March 9, 2013; accepted March 25, 2013.

\section{Author information}

Michele Carriero, Dipartimento di Matematica e Fisica "Ennio De Giorgi”, Università del Salento, Italy.

E-mail: michele.carriero@unisalento.it

Antonio Leaci, Dipartimento di Matematica e Fisica "Ennio De Giorgi”,

Università del Salento, Italy.

E-mail: antonio.leaci@unisalento.it

Franco Tomarelli, Dipartimento di Matematica "Francesco Brioschi",

Politecnico di Milano, Italy.

E-mail: franco.tomarelli@polimi.it 